\title{
Design of life-size haptic environments
}

\author{
Yoky Matsuoka \\ Carnegie Mellon University \\ Pittsburgh, PA 15213 \\ yoky@cs.cmu.edu \\ Bill Townsend \\ Barrett Technology, Inc. \\ Cambridge, MA 02141 \\ wt@barrett.com
}

\begin{abstract}
Brake-actuated haptic devices, a subset of passive haptic devices, are not yet common, so their capabilities and limitations are only superficially understood. In this paper, we identify an optimal kinematics, and introduce a prototype of a life-size brake-actuated haptic device. With the new device, we conducted experiments with human subjects to evaluate the efficacy of passively created virtual environments. We identified key performance drawbacks that modern robotics theories fail to anticipate and overcome, and investigated design methods that can avoid such drawbacks.
\end{abstract}

\section{Introduction}

Motor-actuated devices are widely used in haptics research and advanced teleoperator masters - capable of creating a rich set of virtual environments in the former and recreating physical environments in the latter. Motors, however, introduce the risk of a device striking or overpowering users within reach. With rare exception, safety concerns limit motor-actuated haptic devices to small workspaces.

The replacement of energetic servo-motors with passive actuators such as brakes improves the inherent safety of the system, and expands the range of motion while still allowing us to simulate virtual objects (i.e. to constrain the user's motion inside an object). Safety is essential for acceptance in new applications such as medical procedures, quantitative rehabilitation, advanced exercise training, and entertainment. Even if the device experiences a power, hardware, or software failure, a brake-actuated device is inherently incapable of exceeding the kinetic energy that a user supplies in each motion. Using currently available engineering technology, the workspace of brake-actuated haptic devices can expand to cover whole-body movements without risking a user's safety. With the expanded workspace, a variety of large movements can be trained and quantitatively analyzed.

In this paper, we describe a novel design of a brake-actuated device, and present theoretical and experimental comparisons to active devices. Theoretically, we demonstrate that brake-actuated devices surprisingly require at 
Figure 1. The brake-actuated spherical haptic device we constructed.

least one prismatic joint in order to produce the simplest environments such as viscous fields. A key difference from active devices is that brakes applied at different joints cannot be added vectorially. As a result, it is difficult to prevent the user from penetrating into an object, and at the same time allow motion in collision free directions ("sticky walls" problem discussed below).

In experiments with our prototype device, we evaluated the machine's efficacy with human subjects, exploring various force fields, including damping, stiffness, inertia, and shape (concave, convex) of virtual objects. Analysis confirms the anticipated problems. Relying on the characteristics of human motor perception, we propose a technique to overcome the device limitations.

\section{Prototype Design of an Optimal Human-Scale Brake- Actuated Haptic Device}

In 1995, we set out to design and build the best haptic prototype under the following constraints:

1. Life size - workspace size on the order of cubic meters, forces on the order of tens of kilograms, and fully spatial 6 degrees of freedom (DOF) kinematics.

2. Safe - passive actuation only.

3. Economically practical - with today's mechanisms and materials.

Analysis led us uniquely to the device illustrated in Figures 1, with brakeactuated spherical kinematics for regional motions terminating at an unactuated 3 -axis wrist that supports a user hand grip.

In our analysis of the machine design, we begin by defining key terms. "Servo-actuation" is the ability to modulate joint torques under computer control in order to create the haptic force field that a user experiences. "Energetic servo-actuation" is capable of channeling power from an unlimited external source of energy into the joint torques. "Passive servo-actuation" is physically incapable of channeling power from any external source into the joint torques. "Brake servo-actuation" is a subset of passive servo-actuation that uses servobrakes and does not rely on other passive mechanisms. "Sticky-walls" are 
unique to brake-actuated haptic devices, where a collision with a wall traps the user behind the wall. There are workarounds, as we discuss in this paper.

Ruling out energetic actuation for its life-threatening safety concerns leaves only passive actuation. One technique was explored by Troccaz et al. [1] who used overrun clutches to brake joint movements. These overrun clutches were not servo-actuated and the applied torque purely depended on the joint angles and speed. Another technique for controlling torques passively was through steerable joints. Substantial progress has been made in building a wide array of steerable robotic joints, collectively known as Programmable Constraint Machines (PCMs) and embodied in cobots [2],[3]. These PCMs have the fundamental advantage of eliminating "sticky walls" by actively aligning the constraint direction with the desired motion. Their disadvantage is the sizable side-slip that traction devices exhibit with existing designs and material technology. That disadvantage however may be overcome when new materials become available in the future.

\subsection{Kinematic Design Considerations}

Our approach to expanding the safe use of haptics to life-size was to replace the motors driving each joint axis of a device with similarly sized servo-brakes. We wish to set the kinematics of the robot in such a way that the end point only opposes an externally imposed motion, so to satisfy,

$$
v^{T} f=v^{T}(-c v)=-c v^{2}<0
$$

where $v$ is the end-point velocity vector, $f$ is the force vector opposing the external motion, and $c$ is a constant. Given that the Jacobian matrix, $J$, is invertible and relates endpoint velocities to joint forces $\tau$ and velocities $\theta$, we can express (1) as

$$
v^{T} f=\theta^{T} \quad J^{T}\left(J^{T}\right)^{-1} \tau=\theta^{T} \quad \tau<0
$$

For energetically actuated robots, (2) is sufficient because terms from each joint $i$ can simply add up vectorially as long as the entire sum is less than zero for all joints $n$ :

$$
\theta^{T} \tau=\sum_{i}^{n} \dot{\theta}_{i} \tau_{i}<0
$$

However, if every joints in a robot are actuated by brakes, none of the joints can produce any active force, thus,

$$
\theta_{i} \tau_{i}<0
$$

for each joint $i$, making (2) invalid for brake actuated devices.

The goal is to control forces in the Cartesian space of the user. So a logical first concept is a Cartesian machine with three orthogonal prismatic joints, such as employed on vertical milling machines. For each Cartesian joint $i$,

$$
\dot{\theta}_{i} \tau_{i}=\dot{\theta}_{i}\left(J^{T} f\right)_{i}=\dot{\theta}_{i}\left(J^{T}(-c v)\right)_{i}=\dot{\theta}_{i} j_{i}^{T}(-c J \dot{\theta})=-c \dot{\theta}_{i} j_{i}^{T} \sum_{i}^{n} j_{i} \dot{\theta}_{i}
$$


for all $n$ joints in the robot, where $j_{i}$ is the $i$ th row of $J$. When all joints are orthogonal as in the Cartesian coordinates,

$$
\dot{\theta}_{i} \tau_{i}=-c \dot{\theta}_{i} j_{i}^{T} \sum_{i}^{n} j_{i} \dot{\theta}_{i}=-c \dot{\theta}_{i}^{2} j_{i}^{T} j_{i}<0
$$

Therefore, a Cartesian machine with three orthogonal prismatic joints can oppose an externally imposed motion with brakes. In fact, (6) is true for any of a large number of mathematically orthogonal coordinate frames. But realistically, robotic orthogonal kinematic arrangements are limited to Cartesian, cylindrical, and spherical with spatial mechanisms listed in Table 1.

\begin{tabular}{||c|c|c||}
\hline \hline Kinematic Arrangement & No. of Revolute Joints & No. of Prismatic Joints \\
\hline Cartesian & 0 & 3 \\
\hline Cylindrical & 1 & 2 \\
\hline Spherical & 2 & 1 \\
\hline \hline
\end{tabular}

Table 1. Spatial mechanisms for orthogonal kinematics

Unfortunately, orthogonal kinematics cannot be reasonably constructed without including at least one prismatic joint. In serial-link robotics, kinematic structures with prismatic joints tend to be more massive for a given work volume than purely revolute structures. A massive structure may be acceptable for a CNC machine in order to achieve the high stiffness needed to cut through metal precisely, but the accompanying inertia and friction, each of which degrade backdrivability, is not acceptable for haptic devices.

So why not build a fully revolute brake-actuated haptic device, and eliminate all prismatic joints? In fact, most serial-link robots do just that, abandoning orthogonal kinematics without penalty [4]. For non-orthogonal kinematics, the summation in (6) does not simplify but rather expands to three independent terms (for a 3 DOF system) as:

$$
\dot{\theta}_{i} \tau_{i}=-c \dot{\theta}_{i} j_{i}^{T} \sum_{i}^{n} j_{i} \dot{\theta}_{i}=-c \dot{\theta}_{i}^{2} j_{i}^{T} j_{i}-c \dot{\theta}_{i} \dot{\theta}_{j} j_{i}^{T} j_{j}-c \dot{\theta}_{i} \dot{\theta}_{k} j_{i}^{T} j_{k}<0
$$

Because there are conditions for joint velocities that make (7) to be greater than 0 (i.e. for any $\left.\dot{\theta}_{k}<-\left(\frac{\dot{\theta}_{i}^{2} j_{i}^{T} j_{i}+\dot{\theta}_{i} \dot{\theta}_{i} j_{i}^{T} j_{i}}{\dot{\theta}_{i} j_{i}^{T} j_{k}}\right)\right)$, non-orthogonal robots cannot always oppose an externally imposed motion with brakes.

As an example, Figure 2 shows a non-orthogonal kinematics. The proposed straight motion could be opposed using an energetic device, but not for a brake-actuated device; the motion cannot proceed from $\mathrm{A}$ to $\mathrm{AB}$ nor from $\mathrm{BC}$ to $\mathrm{C}$, but it is permissible from $\mathrm{AB}$ through $\mathrm{B}$ to $\mathrm{BC}$. These permissible areas shift and rotate even for straight-line arm motions, so that most trajectories through the workspace cannot be supported.

\subsection{Prototype with Spherical Kinematics}

Given that we cannot build a simple all-revolute, brake-actuated device, we settle for one of the three orthogonal kinematic structures of Table 1. And 
Figure 2. An illustration of how brake-actuated devices fail to create simple environments such as viscous fields. For a brake-actuated device, Regions A and $\mathrm{C}$ are impossible to attain.

given that revolute joints are preferable over prismatic joints, we chose the option with only one prismatic joint - spherical kinematics.

The prototype is comprised of backdrivable cable drives free of backlash and friction. The revolute joints are on intersecting yaw and pitch axes with 180 and 90 degrees of joint range respectively. A cable drive is also used to drive the prismatic axis, with 1.1 meters of active stroke. All three axes mutually intersect at the kinematic center of the device and all three drives are terminated at one of three servo-controlled magnetic particle brakes. A high-resolution optical incremental encoder is mounted on the free shaft of each servo-brake. An unpowered and unsensored three-axis handle supports the user's grip at the end of the prismatic link. The machine allows for large, sweeping motions from its user with loads up to $50 \mathrm{~kg}$.

The control of the three particle brakes is done via current amplifiers and an I/O board with encoder counters mounted on the bus of a standard Pentium PC running Windows 95. The machine controller converts the encoder readings to Cartesian coordinates and can respond to a user's musculo-skeletal changes in force, position, velocity, acceleration, power, work, and range of motion in real time. LabView graphical interface software allows the user to specify the training variables in a simple manner. A foot pedal is installed within the user's workspace to make fine adjustments or to send commands during training without stopping the motion.

Finally, to prove that the workspace can provide continuous control free of the type of discontinuities predicted for the all-revolute concept, we attached a weight at the end tip of the robot and dropped it in a viscous field as shown in Figure 3. The weight fell in a straight line with very little variation from a perfect downward trajectory, while the prismatic joint changed the velocity direction.

\section{Experimental Evaluation of Brake-Actuated Virtual Environments}

With the spherical life-scale device we designed and constructed, we now explore whether an effective virtual environment can be constructed. Previously, 
Figure 3. We attached a weight at the end of the robot and dropped it in the force field. The weight dropped straight in the Cartesian coordinates while one of the joints changed the velocity direction.

Figure 4. Left: The energetically actuated robot (PHANToM) with a virtual ball overlayed. Middle: Our brake actuated robot with a virtual ball. Right: The simulated environment.

purely dissipative devices were used only to damp human limb movements. To suppress intentional tremor, Rosen and Baiges [5] have developed a tabletop brake-actuated device. It has been demonstrated that mechanical damping on effected joints reduces the amplitude of tremor while allowing slower voluntary movements [6]. The same research group produced viscous joystick [7] and wearable [8] devices to suppress tremor.

We are interested in taking a step further to create a rich set of virtual environments with virtual objects and movement guidance paths. Here, we examine virtual objects with three different force characteristics, namely inertial, viscous, and stiff forces, by conducting simple experiments with human subjects. Inertial, viscous, and stiff forces are proportional to the acceleration, velocity, and position of the machine handle respectively: $F=I a, B v$, or $K\left(x-x_{0}\right)$, where $I$ is a inertial constant, $B$ is a damping constant, and $K$ is a stiffness constant. We are interested in understanding how each force field affects the hand movement, and whether these force fields can prevent movements into a certain region (i.e. inside objects). To compare with equivalent objects created by an energetically actuated device, we used PHANToM to also produce inertial, viscous and stiff virtual objects, as shown in Figure 4.

Four human subjects participated in this study. The computer screen displayed a visual feedback of their hand location in the workspace. There were two targets within the workspace, and subjects were instructed to move from 
Figure 5. Left: Hand trajectories in baseline, viscous and inertial force fields. Hand goes through the virtual object for both active and passive environments. Top Right: Baseline velocity profiles. Right Bottom: Velocity profiles with a viscous object.

one target to another with a sound cue. For the energetically actuated device, the movement was $10 \mathrm{~cm}$ long and had to be executed within 650 milliseconds. For the brake-actuated device, the movement was $100 \mathrm{~cm}$ long and was executed within 1.20 seconds. To establish a baseline movement for all subjects, we first recorded ten hand movements for each subject for each machine without any virtual objects in the workspace. The baseline movements were straight between two points, and their velocity profiles had a single bell-shaped curve for both devices.

Once the baseline movements were established, we placed a virtual sphere in the baseline hand path as shown in Figure $4(2.5 \mathrm{~cm}$ radius for the energetic device and $25 \mathrm{~cm}$ for the brake-actuated device). The virtual object was introduced unexpectedly, and visual feedback was suppressed to assure that subjects did not alter their movement due to the visual information.

First, we studied viscous objects placed in the middle of the hand path. With low viscosity ${ }^{1}$, subjects continuously executed straight movements through the virtual objects for both devices, as shown in Figure 5. The effect of the viscous objects was obvious in the velocity profiles compared to the baseline profiles; the velocities were reduced while the hand was in the object, and resumed to finish the original bell-shape curve after the hand exited the object. Because the end-point moving direction is always consistent with the velocity vector, both energetic and passive devices produced viscous force fields accurately. However, this was not true for virtual objects with inertial force fields. When the end-point movement was decelerating, the brake-actuated robot could not produce assistive force as for the energetic device. As a result, accurate inertial force fields could not be created with brake-actuated devices. In both devices, viscous and inertial objects acted as a tool to slow down movements but the hand trajectories were not deviated from the intended paths.

As a last set of force fields, we created an object with stiff force fields in

\footnotetext{
${ }^{1}$ When the viscosity was set high, control instability was experienced for both devices when the control loop frequency was $1 \mathrm{kHz}$.
} 
Figure 6. Hand paths while interacting with objects with stiff force fields. Using the energetic robot, hand paths were diverted at the surface of the object.

the hand path. Stiff objects apply forces that are proportional to the distance from the boundary of the object. The deeper the penetration, the stronger the force applied. With the brake-actuated device, the subject's hand still projected a straight movement, as shown in Figure 6. When the stiffness was set low, the hand went through the object, and when the stiffness was high the hand stopped just inside the object. When the hand was stopped inside the object, the resistive force was strong for all moving directions, causing subjects to experience "sticky walls".

In contrast, when the energetically actuated device created stiff objects, the hand changed its course. The hand penetrated the object slightly then slipped off to the side of the object before continuing to the target. This type of movement deviation was observed for a variety of stiffness, and subjects perceived a wide range of object hardness corresponding to the stiffness of the object. This is the behavior we want to reproduce using brake-actuated device, but we must first get around the "sticky walls" problem.

As a first attempt to remove the sticky walls, the velocity vector orientation was monitored throughout the program. When the hand penetrates the object, ideally, the hand should be freed away from the object surface. Therefore, we set a condition to turn off the forces if the velocity vector did not point into the object. This technique was effective at a high velocity, but singularities occurred when the velocity vector did not specify the moving direction (i.e. velocity equaled to zero). At singularities, without predicting the next moving direction prior to the move, brakes could never respond correctly.

Furthermore, monitoring the velocity vector did not cause any movement diversion that we desire to produce. How can we make an environment that imposes a restriction into a selected region? As a way to constrain movements into a certain path, we could eliminate a joint from the device and make a two DOF robot. If one of the brakes on the device is locked, the simulated environment should be reduced to a plane as if eliminating a joint. If two of the brakes are locked, the environment should be further restricted to a line. Even though these planes and lines are restricted to the machine constraints, if there are ways to use this locking technique with respect to virtual objects, we may be able to divert movements using purely dissipative devices.

To combine the locking technique with virtual objects, an object contact 
Figure 7. By aligning machine axes with exit plane of the virtual object, passively created stiff objects could divert hand paths as shown on the right.

point was first defined as illustrated in Figure 7 . We defined a contact velocity vector as the hand velocity at contact. In addition, we defined a tangential plane with respect to the object at the contact point. This tangential plane is a collection of tangential vectors, defined as exit vectors, that the hand movement should follow immediately after contacting the object. Friction cone was defined to be 60 degrees wide. When the contact velocity vector was within the friction cone, then the movement was not deviated. If the contact velocity vector was outside of the friction cone, and any of the exit vectors was closely aligned with one of the machine axes, then an appropriate joint was locked to move the hand along the exit plane. After the hand deviation was completed, the joint was unlocked. Figure 7 shows an example where hand trajectories were diverted from an object using our brake-actuated device.

We tested various movement cases with a virtual sphere, and found that we cannot consistently align the machine axes with the exit plane. As a way to overcome this issue, we created a workaround that utilizes the discrepancy in human visual and proprioceptive perception. When humans are given perturbed visual information, their motor behavior adjusts to match the visual input. Due to this perceptual gap, some haptic inaccuracy may be tolerated if the visual feedback is consistent. In order to use this gap, the subject's movements were projected for 50 milliseconds in advance using the movement velocity profiles. This projection estimated the contact point with the object and the exit vector. If the projected exit vector did not align well with the machine axes, the closest point along the movement trajectory with an alignment was searched. When the point was found, the object was moved to that location and the visual feedback sped up or slowed down proportionally to match the new object location. With this search algorithm, we were able to divert a hand movement on a virtual sphere for all cases.

A limitation with this technique was the shape of the object. A sphere was a convenient example because the tangential plane was always outside of the object. However, problems arose when the entire tangential plane was in the object (i.e. concave objects). Therefore, concave objects had spots that could not be declared as a contact point. The same search algorithm was 
implemented to find the nearest non-concave points, but the technique was ineffective for large concave surfaces.

\section{Conclusions}

Using the optimal life-scale brake-actuated haptic device, we created a large and safe haptic environment. We were able to create virtual objects and guide movement path in this new environment, but the non-energetic nature of the brakes imposed limitations on the virtual environments. Perhaps, the most exciting part of this investigation was the development of algorithms utilizing human movement and perceptual information, but there were still limitations that we could not eliminate.

We intend to improve the design by adding force sensors in the handle. If we could detect the movement direction before the device joints starts moving, then we may be able to eliminate the sticky walls. Furthermore, we plan to add weights and springs to produce negative inertial and stiff forces for some applications.

\section{Acknowledgments}

We would like to thank Dr. Pierre Dupont for his insightful inputs.

\section{References}

[1] Troccaz J, Lavallee S, Hellion E 1993 Padyc: A passive arm with dynamic constraints. Int'l. Conf. on Advanced Robotics, pp.361-366.

[2] Colgate J E, Peshkin M A, Wannasuphoprasit W 1996 Nonholonomic haptic display. Proc of the IEEE Int'l Conf. on Robotic and Automation.

[3] Peshkin M A Colgate J E 1999 Cobot architecture. IEEE Tans. Robotics and Automation.

[4] Townsend W T 1988 The effect of transmission design on force - controlled manipulator performance. $\mathrm{PhD}$ thesis, Massachusetts Institute of Technology

[5] Baiges I, Rosen M 1989 Development of a whole-arm orthosis for tremor suppression. Proc. of the 12th Annual Conf. on Rehab. Technology. p.290-1.

[6] Aisen M L, Arnold A, Baiges I, Maxwell S, Rosen M 1993 The effect of mechanical damping loads on disabling action tremor. Neurology. 43(7):1346-50.

[7] Beringhause S, Rosen M, Huang S 1989 Evaluation of a damped joystick for people disabled by intention tremor. Proc. of the 12th Annual Conf. on Rehab Technology. p.41-2.

[8] Kotovsky J, Rosen M J 1998 A wearable tremor-suppression orthosis. J. of Rehabilitation Research and Development. 35(4):373-387. 